\title{
Complete deltoid resection in early childhood without muscle transfer results in normal shoulder function at long-term follow-up: a case report
}

\author{
Annie Arteau ${ }^{1}$, Franziska Seeli ${ }^{2}$ and Bruno Fuchs ${ }^{2^{*}}$
}

\begin{abstract}
Background: Musculoskeletal tumors involving the deltoid muscle and necessitating its complete resection are rare. The function after complete deltoid resection is reported to be limited, and several authors consider muscle transfer to improve shoulder motion. However, it still remains unclear whether such transfer adds function. To the best of our knowledge, all reports on complete deltoid resection refer to adult patients, and it is unknown what function results after deltoid resection in childhood. The remaining muscles may have the potential to compensate for the loss of deltoid function.
\end{abstract}

Case presentation: Here we report the case of a 5-year-old white boy with complete (isolated) deltoid muscle resection in infancy for a large aggressive soft tissue tumor. No reconstructive procedure or muscle transfer was performed at the time of index surgery. Pathology revealed an angiomatoid fibrous histiocytoma. His postoperative course was uneventful. At 11 years of follow-up, he remained disease-free and had excellent shoulder function, including normal range of motion.

Conclusions: This report implies that major muscles such as the deltoid can be resected in a child without compromising long-term function. Therefore, a muscle transfer at index surgery is probably not necessary.

Keywords: Deltoid resection, Shoulder function, Pediatric

\section{Background}

Most of the knowledge related to deltoid dysfunction stems from patients with non-neoplastic pathologies such as brachial plexus injuries, axillary nerve palsy secondary to shoulder luxation, iatrogenic nerve or muscle lesions, or after poliomyelitis $[1,2]$. Loss of anterior deltoid function had been historically associated with impairment of glenohumeral function and chronic pain [1-5]. When associated with a rotator cuff tear, impairment is higher, nerve reconstruction or muscle transfer success is lower $[1,3,4,6]$.

Muscle or nerve transfer or graft for deltoid insufficiencies of any cause is associated [1,3-6] with variable

\footnotetext{
* Correspondence: bruno.fuchs@uzh.ch

${ }^{2}$ Sarkomzentrum UZH, University of Zurich, Forchstrasse 340, CH-8008 Zurich, Switzerland

Full list of author information is available at the end of the article
}

success rates. Only a few authors reported a complete range of motion with complete deltoid palsy without any reconstructive procedure [6]; they suggested that sufficient function could be obtained to allow full duty work.

Complete resection of the deltoid muscle for musculoskeletal tumors is rarely performed $[7,8]$. In the tumor literature, where the loss of the deltoid muscle can be regarded as an isolated lesion with a normal rotator cuff, it is thought that partial resection of the deltoid muscle may result in normal shoulder function $[8,9]$, whereas complete resection compromises its function. Because of the purported limited function, consideration is often given to the addition of a latissimus dorsi, a trapezius, or a pectoralis major transfer to potentially improve the functional outcome $[10,11]$. 


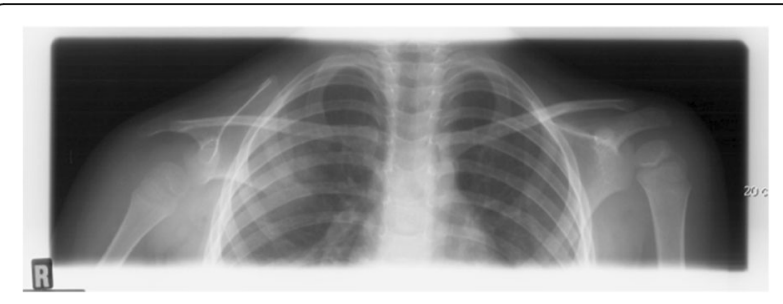

Fig. 1 At initial presentation, the radiographs showed an inferior subluxation of the right shoulder

Therefore, there is continuing debate on whether a muscle transfer may improve shoulder function after complete deltoid resection. Some authors recommend muscle transfer to improve shoulder motion or strength, whereas others recommend muscle transfer solely for tissue coverage because they believe it adds little to the improvement of shoulder function. Of interest, all this literature reports on adult patients and it is unclear whether this also applies to children, who may have a greater adaptability and potential compensation for their shoulder muscle function.

\section{Case presentation}

A 5-year-old white boy presented with a 1-year history of right shoulder pain and asymmetrical right scapula. His shoulder range of motion was preserved as well as function. Amyotrophy was initially suspected but excluded based on normal neurologic investigation. There was no significant past medical or family history. Imaging revealed a persistent subluxation of the humeral head of unclear genesis (Fig. 1). Subsequent investigations by computed tomography (CT) scan and MRI showed a $5 \times 3.5 \times 2.5 \mathrm{~cm}$ soft tissue mass with an aggressive appearance within his deltoid muscle but without involvement of his brachial plexus. An initial biopsy failed to determine the exact nature of the lesion but revealed an aggressive biology. Based on the clinical and aggressive appearance of the lesion on imaging, the remaining diagnosis was a malignancy. Consequently, a complete deltoid resection was performed on this child because of the infiltrative pattern of the tumor through the entire muscle (Fig. 2). Surgery included a straight lateral, vertical incision from his acromion to the deltoid insertion (Fig. 3e). The posterior border of his deltoid muscle was prepared and the axillary neurovascular bundle identified. Muscular detachments from the deltoid insertion and then his acromion followed, before his entire muscle was mobilized together with the tumor. No reconstructive procedure was performed at the time of index procedure or later.

Histological analysis of the tumor revealed an angiomatoid fibrous histiocytoma. At the time of diagnosis, this lesion was known as angiomatoid malignant fibrous histiocytoma because initially its metastatic potential was considered much higher than it is now. Angiomatoid fibrous histiocytoma is a low grade lesion with a $1 \%$ metastatic rate. Complete surgical resection is usually curative. No adjuvant therapy was performed. His postoperative course was uneventful. Over the first few weeks, physical therapy (PT) assisted in mobilizing his shoulder joint. Six months postoperatively, the child demonstrated near complete range of motion of his right shoulder. Over the years, the growing child participated in normal physical activities and maintained a symmetric range of motion. Ten years after the complete deltoid resection, he is free of disease and his shoulder function remains excellent. When compared to his left side, the range of motion of his right shoulder is symmetrical and normal (Fig. 3). Normal rotator cuff muscles and good centralization of the humeral head were observed without degenerative changes on a recent MRI at 11 years of follow-up (Fig. 4). His Musculoskeletal Tumor Society Score (MSTS; a score widely used in orthopedic oncology; it addresses pain, function, emotions, hand positioning, manual dexterity, and lifting ability) for upper extremity is excellent (93.3\%), his Constant Shoulder Score (a score widely used in shoulder surgery; it addresses pain, activity of daily living, arm positioning, range of motion, and abduction power) is $75.8 \%$ on his

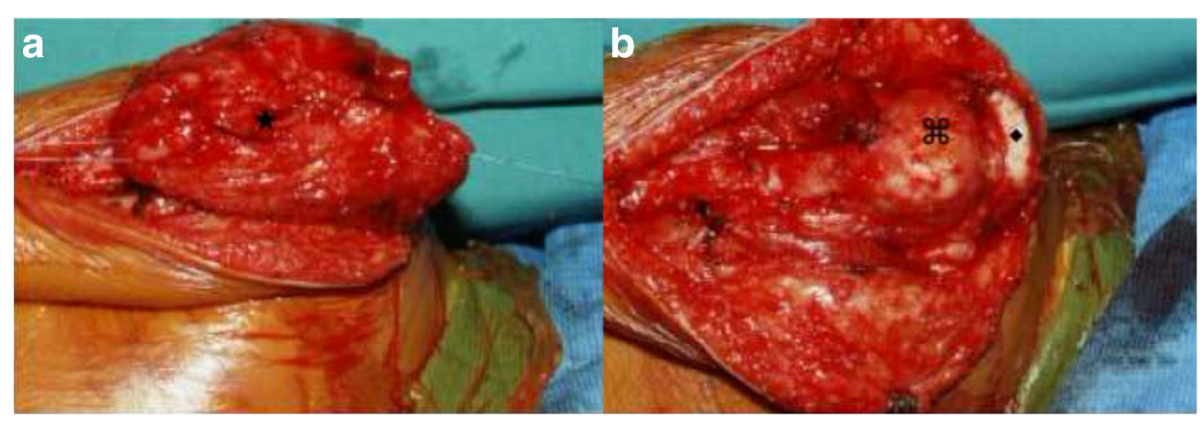

Fig. 2 Intraoperative pictures showing infiltration of the muscle and subcutis. a The tumor within the deltoid muscle ( $\star \star)$. After deltoid muscle resection, the humeral head $(\mathscr{H})$ and the acromion $(\bullet)$ can be seen $(\mathbf{b})$ 


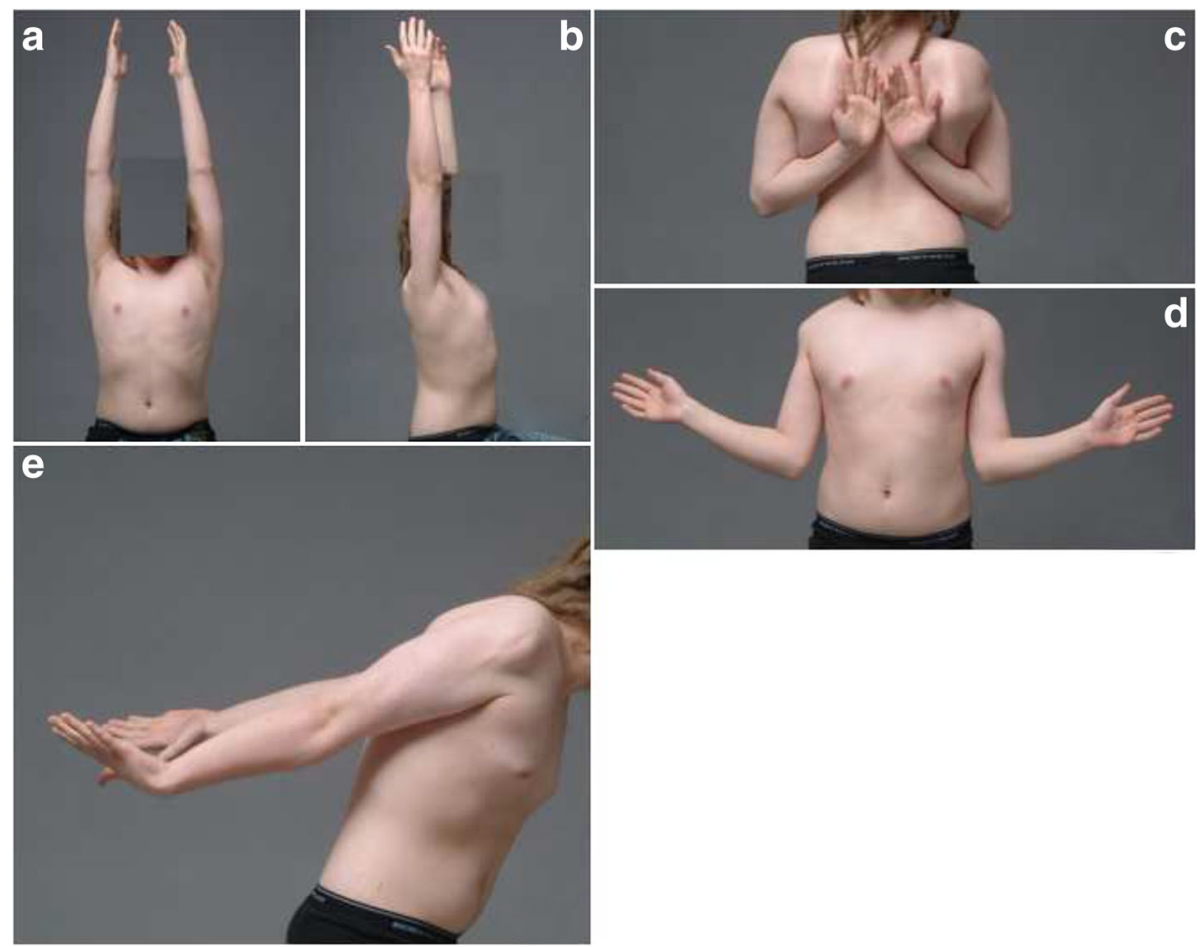

Fig. 3 Normal range of motion 9 years after surgery. Our patient is now 16-years old. Abduction in the scapular plane (Fig. 3a), forward elevation (Fig. 3b), internal rotation (Fig. 3c), external rotation (Fig. 3d), and extension are normal (Fig. 3e)

right side and $80 \%$ on his left side. Patient selfassessment of his shoulder function (subjective shoulder value) is excellent (95\%) with only slight weakness noted when heavy objects are carried. Despite excellent function reported, a slight difference to the opposite side is observed. Objectively, his shoulder strength in forward elevation is $54.6 \mathrm{~kg}$, internal rotation is $20.8 \mathrm{~kg}$, and external rotation is $15.8 \mathrm{~kg}$ compared to $76 \mathrm{~kg}, 23.4 \mathrm{~kg}$, and $24.1 \mathrm{~kg}$ respectively for his contralateral shoulder. MRI revealed an intact rotator cuff without atrophy of his supraspinatus, infraspinatus, and subscapularis muscles (Fig. 4).

\section{Discussion}

We can only speculate about the reasons why there is normal shoulder function despite the complete removal of the deltoid muscle. In the normal shoulder, a combination of muscles generates a complex movement: anterior deltoid, supraspinatus, coracobrachialis, long head of biceps brachii, and clavicular portion of pectoralis major act together to provide power and movement in forward flexion. Abduction in the scapular plane is generated by the middle portion of the deltoid, supraspinatus, and a coupled force between subscapularis and infraspinatus $[12,13]$. A selective bloc of the suprascapular or axillary

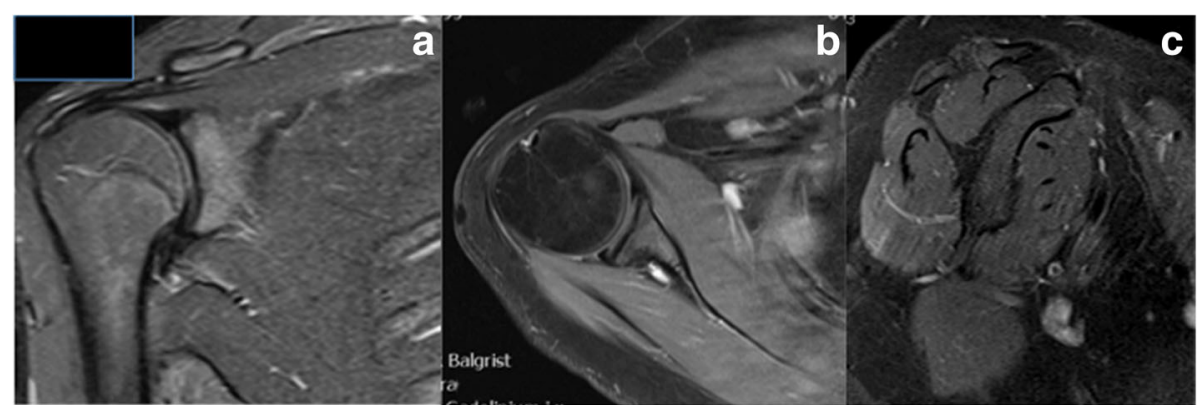

Fig. 4 Magnetic resonance imaging (Fig. 4a frontal plane; Fig. 4b axial plane; Fig. 4c parasagittal plane) of the patient's shoulder at 11 years of follow-up shows normal rotator cuff muscles without atrophy and fatty streaks, but the absence of the deltoid muscle 
nerve showed that the deltoid muscle provides $50 \%$ of the power for forward elevation and abduction in the plane of the scapula. Supraspinatus and infraspinatus generate almost $50 \%$ of the torque; infraspinatus acts mostly above 90 degrees of abduction. Electromyographic studies [14] showed a silent signal before 90 degrees of abduction. In the absence of deltoid and rotator cuff muscles, other muscles cannot generate enough torque to overcome gravity but act as a movement generator $[12,13]$. Of interest, our patient showed excellent strength in abduction in the plane of the scapula without deltoid muscle. In addition, an MRI of his shoulder at 11-years follow-up showed normal shoulder muscles, without intraarticular degenerative changes. Although up to $20 \%$ of the patients with a complete deltoid dysfunction may achieve full (passive) range of shoulder motion $[6,15]$, our patient also had very good strength. Altogether, this implies that the other shoulder muscles can compensate for the loss of deltoid function after its complete removal in early childhood. Isolated deltoid resection is a rare procedure, even more in the pediatric population, and consequently it is difficult to make recommendations regarding the need for reconstruction or not based on this specific case. On the other hand, the excellent long-term follow-up of this patient with an annual physical examination and medical photography have showed that excellent function and strength remain over years without reconstruction.

\section{Conclusions}

To the best of our knowledge, this is the first report of complete deltoid resection without muscle transfer in a child, resulting in a full range of motion, excellent strength, and excellent function of the shoulder at longterm follow-up. The remaining shoulder muscles have the potential to compensate for the loss of deltoid function in very young children thereby avoiding the need for complex procedures like pedicled latissimus muscle flap or trapezius transfer unless needed for soft tissue coverage.

\section{Acknowledgement \\ None.}

\section{Funding}

The authors (AA, BF, FS) declare that they have not received an external source of funding for the present study.

\section{Availability of data and materials}

The data presented in this case report are the original patient's data. Therefore, the authors will not share it in an additional file. The tests used in this case report, namely Constant Shoulder Score and MSTS, are internationally approved, reliable, and validated. The tests are freely available and accessible for any scientist interested.

\section{Authors' contributions}

AA examined the patient, analyzed and interpreted the patient's data, and wrote the first draft of the manuscript. FS acquired the patient data and was involved in drafting the manuscript. BF provided the concept and study design, analyzed and interpreted the data, and rewrote the manuscript. All authors read and approved the final manuscript.

\section{Competing interests}

The authors declare that they have no competing interests.

\section{Consent for publication}

Written informed consent was obtained from the patient for publication of this case report and any accompanying images. A copy of the written consent is available for review by the Editor-in-Chief of this journal.

\section{Ethics approval and consent to participate}

The case was reviewed and waived by the leader of orthopedic research at Balgrist University Hospital, Zurich. Approval from the cantonal ethics committee of Zurich was not required and therefore not obtained.

\section{Author details}

'Hôtel Dieu de Québec, 11 Côte du Palais, Quebec G1R 2J6, Canada.

${ }^{2}$ Sarkomzentrum UZH, University of Zurich, Forchstrasse 340, CH-8008 Zurich, Switzerland.

Received: 17 March 2016 Accepted: 3 November 2016

Published online: 14 January 2017

\section{References}

1. Resch H, Povacz P, Maurer H, Koller H, Tauber M. Pectoralis major inverse plasty for functional reconstruction in patients with anterolateral deltoid deficiency. J Bone Joint Surg Br. 2008;90:757-63.

2. Groh Gl, Simoni M, Rolla P, Rockwood CA. Loss of the deltoid after shoulder operations: An operative disaster. J Shoulder Elbow Surg. 1994;3:243-53.

3. Forsythe B, Lavery KP, Warner JJP. Acromionectomy and deltoid deficiency: a solution. Clin Orthop Relat Res. 2009;467:1334-40.

4. Itoh Y, Sasaki T, Ishiguro T, Uchinishi K, Yabe Y, Fukuda H. Transfer of latissimus dorsi to replace a paralysed anterior deltoid. A new technique using an inverted pedicled graft. J Bone Joint Surg Br. 1987;69:647-51.

5. Chebli CM, Murthi AM. Deltoidplasty: outcomes using orthobiologic augmentation. J Shoulder Elbow Surg. 2007:16:425-8.

6. Dehne E, Hall RM. Active shoulder motion in complete deltoid paralysis. J Bone Joint Surg Am. 1959:41-A:745-8.

7. Khruekarnchana P, Suzuki K, Fukuda H. Shoulder function after subtotal excision of the deltoid muscle and of the deltoid and infraspinatus muscles in extra-abdominal desmoid tumor: a long-term follow-up in two cases. J Shoulder Elbow Surg. 2004;13:119-22.

8. Markhede G, Monastyrski J, Stener B. Shoulder function after deltoid muscle removal. Acta Orthop Scand. 1985;56:242-4.

9. Mimata Y, Nishida J, Gotoh M, Akasaka T, Shimamura T. Limb function after excision of a deltoid muscle sarcoma. J Shoulder Elbow Surg. 2013;22:e1-5.

10. Muramatsu K, Ihara K, Tominaga Y, Hashimoto T, Taguchi T. Functional reconstruction of the deltoid muscle following complete resection of musculoskeletal sarcoma. J Plast Reconstr Aesthet Surg. 2014;67:916-20.

11. Muramatsu K, Ihara K, Doi K, Yoshida K, Iwanaga R, Hashimoto T, Taguchi T. Functional neuro-vascularized muscle transfer for oncological reconstruction of extremity sarcoma. Surg Oncol. 2012;21:263-8.

12. Howell SM, Imobersteg AM, Seger DH, Marone PJ. Clarification of the role of the supraspinatus muscle in shoulder function. J Bone Joint Surg Am. 1986; 68:398-404

13. Colachis SC, Strohm BR. Effect of suprascauular and axillary nerve blocks on muscle force in upper extremity. YAPMR. 1971;52:22-9.

14. Poppen NK, Walker PS. Forces at the glenohumeral joint in abduction. Clin Orthop Relat Res. 1978;135:165-70.

15. Bonnard C, Anastakis DJ, van Melle G, Narakas AO. Isolated and combined lesions of the axillary nerve. A review of 146 cases. J Bone Joint Surg Br. 1999:81:212-7. 